\title{
Cost-effectiveness of malaria preventive treatment for HIV-infected pregnant women in sub-Saharan Africa
}

Sung Eun Choi ${ }^{* *}$, Margaret L. Brandeau ${ }^{1}$ and Eran Bendavid ${ }^{2,3}$

\begin{abstract}
Background: Malaria is a leading cause of morbidity and mortality among HIV-infected pregnant women in subSaharan Africa: at least 1 million pregnancies among HIV-infected women are complicated by co-infection with malaria annually, leading to increased risk of premature delivery, severe anaemia, delivery of low birth weight infants, and maternal death. Current guidelines recommend either daily cotrimoxazole (CTX) or intermittent preventive treatment with sulfadoxine-pyrimethamine (IPTp-SP) for HIV-infected pregnant women to prevent malaria and its complications. The cost-effectiveness of CTX compared to IPTp-SP among HIV-infected pregnant women was assessed.
\end{abstract}

Methods: A microsimulation model of malaria and HIV among pregnant women in five malaria-endemic countries in sub-Saharan Africa was constructed. Four strategies were compared: (1) 2-dose IPTp-SP at current IPTp-SP coverage of the country ("2-IPT Low"); (2) 3-dose IPTp-SP at current coverage ("3-IPT Low"); (3) 3-dose IPTp-SP at the same coverage as antiretroviral therapy (ART) in the country ("3-IPT High"); and (4) daily CTX at ART coverage. Outcomes measured include maternal malaria, anaemia, low birth weight (LBW), and disability-adjusted life years (DALYs). Sensitivity analyses assessed the effect of adherence to CTX.

Results: Compared with the 2-IPT Low Strategy, women receiving CTX had 22.5\% fewer LBW infants (95\% Cl 22.322.7), 13.5\% fewer anaemia cases ( $95 \% \mathrm{Cl} 13.4-13.5)$, and $13.6 \%$ fewer maternal malaria cases ( $95 \% \mathrm{Cl} 13.6-13.7)$. In all simulated countries, CTX was the preferred strategy, with incremental cost-effectiveness ratios ranging from costsaving to $\$ 3.9$ per DALY averted from a societal perspective. CTX was less effective than the 3-IPT High Strategy when more than $18 \%$ of women stopped taking CTX during the pregnancy.

Conclusion: In malarious regions of sub-Saharan Africa, daily CTX for HIV-infected pregnant women regardless of CD4 cell count is cost-effective compared with 3-dose IPTp-SP as long as more than 82\% of women adhere to daily dosing.

\section{Background}

HIV and malaria are leading causes of disease burden in sub-Saharan Africa; combined, the two diseases cause nearly $20 \%$ of all deaths annually $[1,2]$. While the geographical epicenters of the two diseases are differentmalaria is most common in Central and West Africa, where HIV prevalence is relatively low, and HIV is most prevalent in Southern Africa, where the malaria burden

\footnotetext{
*Correspondence: sungeunc@stanford.edu

1 Department of Management Science and Engineering, Stanford University, Stanford, CA, USA

Full list of author information is available at the end of the article
}

is lower-the overlap is substantial, especially in Eastern Africa, as is the burden of co-infection [3]. Each disease potentially exacerbates the other: HIV-infected adults are at increased risk of complicated and severe malaria and death, while malaria results in increased viral load and may speed disease progression and spread [4].

Malaria is an important cause of morbidity and mortality among HIV-infected pregnant women in co-endemic regions of sub-Saharan Africa, where at least 1 million malaria co-infections occur among pregnant women annually [5]. HIV-infected pregnant women who experience clinical malaria are at increased risk of premature 
delivery, severe anaemia, delivery of low birth weight infants, and death $[6,7]$.

Guidelines currently encourage providing pregnant women with malaria preventive treatment during pregnancy in malaria-endemic countries [8, 9]. Previous guidelines recommended 3 doses of intermittent preventive treatment with sulfadoxine-pyrimethamine (IPTpSP) for HIV-infected pregnant women with CD4 > 350 cells $/ \mathrm{mm}^{3}$ or daily cotrimoxazole (CTX) for women with CD4 < 350 cells $/ \mathrm{mm}^{3}$ [10]. Despite these guidelines, median coverage rate of receiving at least 1 dose of IPTpSP from antenatal clinics in sub-Saharan Africa is $19.0 \%$, which remains markedly lower than the international targets, despite high rates of antenatal clinic attendance (75\% of women in sub-Saharan Africa attend at least twice) $[11,12]$. In analyses of the low coverage of IPTpSP in sub-Saharan Africa, information barriers (unclear guidelines and uncertain messages about timing of IPTpSP in pregnancy), behavioral barriers (irregular antenatal clinic visits), and system barriers [sulfadoxine-pyrimethamine (SP) stockouts and low clinic staffing] emerge as salient [13-18].

In 2014, the World Health Organization (WHO) provided updated guidelines that recommend CTX for pregnant women with severe or advanced HIV clinical disease (WHO stages 3 or 4) or CD4 cell counts below 350 cells $/ \mathrm{mm}^{3}$ and, in contexts where malaria or severe bacterial infections are common, CTX regardless of CD4 cell count or HIV severity [8].

Implementation of the WHO guidelines remains mixed. A cross-sectional analysis of observational data in Malawi found that daily CTX was associated with reduced malaria parasitaemia and anaemia compared to IPTp-SP among HIV-positive pregnant women [19]. However, while CTX was found to be effective in preventing clinical malaria $[20,21]$, long-term efficacy relative to standard IPTp-SP, toxicity, and birth outcomes associated with CTX have not been established in trials [22]. Many national guidelines still recommend a mix of IPTp-SP and CTX because of past WHO guidelines that recommended IPTp-SP, and because imperfect adherence to daily CTX may put women at risk compared with only 3 doses of IPTp-SP [23].

A model-based analysis to assess the effectiveness and cost-effectiveness of daily CTX relative to IPTp-SP among HIV-infected pregnant women in sub-Saharan Africa was performed.

\section{Methods}

An individual-level microsimulation model of malaria among HIV-infected pregnant women was constructed and parameterized to five sub-Saharan countries: Ghana, Kenya, Malawi, Mozambique, and Tanzania. These countries were selected because they are all co-endemic, with wide ranges of HIV and malaria burdens.

Cohorts of 10,000 HIV-infected pregnant women from each country were simulated over a 40 -week pregnancy period. Each woman was characterized by CD4 cell count, antiretroviral therapy (ART) status, and malaria preventive treatment status. Tables 1 and 2 summarizes key model parameters and data sources, further detailed in the Additional file 1, consistent with international model reporting guidelines [24]. Here, an overview of the model's key components is provided.

\section{Treatment strategies}

The Reference Strategy modeled receipt of 2 doses of IPTp-SP when CD4 is above 350 cells $/ \mathrm{mm}^{3}$, and daily CTX when CD4 is below 350 cells $/ \mathrm{mm}^{3}$. The portion of the model population (HIV-infected pregnant women) receiving these preventive options in each country reflected the current IPTp-SP coverage rate in that country and, therefore, represented common practice in many at-risk populations [25]. Three alternative strategies for reducing risk of malaria in pregnant women were simulated in addition to the Reference Strategy: (1) 3 doses of IPTp-SP if CD4 cell count $>350$ and daily CTX if CD4 cell count $<350$, at the current IPTp-SP coverage in the country of interest (3-IPT Low Strategy) [26]; (2) same as the 3-IPT Low Strategy, but at a higher coverage equivalent to current ART coverage rates (3-IPT High Strategy); and (3) daily CTX regardless of CD4 cell count (CTX Strategy).

The 3-IPT Low Strategy reflects the possibility that the current capacity to provide IPTp-SP remains unchanged, but adherence to the policy of providing 3 doses (rather than 1 or 2) is improved. In the 3-IPT High Strategy, we assumed that IPTp-SP and CTX coverage rates would reach the current ART coverage rate of the country. ART coverage rates were used as the target coverage rates for IPTp-SP in this strategy, assuming that malaria prevention services could be provided through the same channels that provide HIV care for pregnant women. In the CTX Strategy, the CTX coverage rate was assumed to be the same as the ART coverage rate: women receiving antenatal HIV care were assumed to receive CTX at the same time, regardless of CD4 cell count. It was assumed that in the CTX Strategy, pregnant women with CD4 cell count $<350$ would be on CTX at the beginning of their pregnancy (assuming they were placed on CTX prior to pregnancy), and those with CD4 cell counts > 350 would receive CTX starting in the second trimester. In the model, pregnant women with CD4 cell counts $>350$ started IPTp-SP in the second trimester and thus received no malaria prophylaxis in the first trimester [27]. 
Table 1 Model parameters

\begin{tabular}{|c|c|c|c|}
\hline Variable & Value & Distribution & Source \\
\hline \multicolumn{4}{|l|}{ Demographic } \\
\hline Malaria prevalence in HIV-infected pregnant women & $31.0 \%$ & $\operatorname{Beta}(3.1,6.9)$ & {$[46]$} \\
\hline CD4 count & 390 & Normal(390,220) & {$[47]$} \\
\hline \multicolumn{4}{|l|}{ Incidence } \\
\hline Relative risk of malaria in HIV-infected pregnant woman & 1.6 & $\operatorname{Gamma}(1.6,1)$ & {$[48]$} \\
\hline CD4 change while on ART & 70 & Normal $(70,100)$ & [49] \\
\hline \multicolumn{4}{|l|}{ Mortality } \\
\hline Neonatal mortality risk due to low birth weight & $6.93 \%$ & Beta $(0.007,0.093)$ & {$[30]$} \\
\hline Annual mortality due to malaria during pregnancy & $0.33 \%$ & Beta $(0.033,9.967)$ & {$[30]$} \\
\hline Annual mortality due to anaemia in pregnancy & $1.0 \%$ & $\operatorname{Beta}(0.1,9.9)$ & {$[30]$} \\
\hline Annual HIV mortality among HIV-infected pregnant women & $0.0161 \%$ & $\operatorname{Beta}(0.16,9.84)$ & {$[50]$} \\
\hline Annual HIV mortality among HIV-infected pregnant women on ART & $0.007 \%$ & $\operatorname{Beta}(0.07,9.93)$ & {$[47,50]$} \\
\hline Relative risk of HIV mortality of CTX while on ART & 0.47 & $\operatorname{Beta}(8,9)$ & {$[35]$} \\
\hline \multicolumn{4}{|l|}{ DALY calculations } \\
\hline Annual discount rate & $3 \%$ & $3 \%$ & [51] \\
\hline Average age (years) & 22.83 & 22.83 & {$[29]$} \\
\hline Life expectancy for women aged 20-24 (years) & 47.24 & Normal $(47,3)$ & {$[32]$} \\
\hline Life expectancy at birth (years) & 57.96 & Normal $(58,3)$ & {$[32]$} \\
\hline Disability weight_-malaria during pregnancy & 0.21 & Normal $(0.21,0.03)$ & {$[31]$} \\
\hline Disability weight_-maternal anaemia due to malaria & 0.06 & Normal $(0.06,0.02)$ & {$[31]$} \\
\hline Disability weight_-low birth weight & 0.11 & 0.11 & {$[33]$} \\
\hline Length of disability (years) — malaria during pregnancy & 0.01 & $\operatorname{Gamma}(2 \cdot 0,0.004)$ & {$[30]$} \\
\hline Length of disability (years) - maternal anaemia due to malaria & 0.06 & Gamma(1.2,0.04) & {$[52]$} \\
\hline Length of disability (years) —low birth weight & 57.96 & $\operatorname{Gamma}(32,1.8)$ & {$[32]$} \\
\hline \multicolumn{4}{|l|}{ Costs (USD 2015) } \\
\hline \multicolumn{4}{|l|}{ Drug cost } \\
\hline Intermittent preventive treatment (per dose) & $\$ 0.20$ & $\operatorname{Normal}(0.2,0.01)$ & {$[30,53]$} \\
\hline Cotrimoxazole, 480 mg twice daily (per year) & $\$ 6.28$ & Gamma(3.9,1.61) & {$[54]$} \\
\hline \multicolumn{4}{|l|}{ Healthcare labor cost ${ }^{a}$} \\
\hline Labor time per IPTp-SP administration (min) & 8.31 & Gamma(13.85,0.6) & {$[30]$} \\
\hline Nurses' monthly cost of labor & 542.76 & $\operatorname{Gamma}(29.3,18.5)$ & {$[30]$} \\
\hline \multicolumn{4}{|l|}{ Household cost } \\
\hline Antenatal care visit direct cost & 0.47 & Gamma $(92.4,0.005)$ & {$[30]$} \\
\hline Antenatal care visit indirect cost & 1.17 & Gamma(146,0.008) & {$[30]$} \\
\hline ART treatment (per year) & $\$ 193.61$ & $\operatorname{Gamma}(2,97)$ & {$[55]$} \\
\hline \multicolumn{4}{|l|}{ Efficacy } \\
\hline \multicolumn{4}{|l|}{ Low birth weight } \\
\hline Relative risk baseline (IPTp-SP 0-1 dose) vs. 2 dose IPTp-SP & 3.25 & Gamma(1.1,2.95) & {$[45]$} \\
\hline Incidence per 1000 women given IPTp-SP 2 doses ${ }^{b}$ & 175 & $(91-222)^{\mathrm{a}}$ & {$[29]$} \\
\hline Relative risk IPTp-SP 3 doses vs. 2 doses & 0.86 & Normal $(0.86,0.21)$ & {$[29]$} \\
\hline Relative risk CTX vs. 2 dose IPTp-SP & 1.16 & Gamma $(1.05,1.1)$ & {$[34,56]$} \\
\hline \multicolumn{4}{|l|}{ Maternal parasitaemia } \\
\hline Relative risk baseline (IPTp-SP 0-1 dose) vs. 2 dose IPTp-SP & 1.4 & Gamma(1.15,1.2) & {$[19]$} \\
\hline Incidence per 1000 women given IPTp-SP 2 doses ${ }^{b}$ & 112 & $(0-359)^{a}$ & [29] \\
\hline Relative risk IPTp-SP 3 doses vs. 2 doses & 0.26 & Normal $(0.26,0.08)$ & {$[29]$} \\
\hline Relative risk CTX vs. 2 dose IPTp-SP & 0.44 & Lognormal $(-1.05,0.7)$ & {$[19,34]$} \\
\hline Relative risk $C T X+A R T$ vs. CTX & 0.38 & Lognormal $(-1.2,0.7)$ & {$[21]$} \\
\hline \multicolumn{4}{|l|}{ Anaemia } \\
\hline Relative risk baseline (IPTp-SP 0-1 dose) vs. 2 dose IPTp-SP & 1.03 & Gamma(0.85, 1.2) & {$[45]$} \\
\hline
\end{tabular}


Table 1 continued

\begin{tabular}{|c|c|c|c|}
\hline Variable & Value & Distribution & Source \\
\hline Incidence per 1000 women given IPTp-SP 2 doses $^{b}$ & 582 & $(333-795)^{a}$ & {$[29]$} \\
\hline Relative risk IPTp-SP 3 doses vs. 2 doses & 582 & Normal(0.96,0.07) & {$[29]$} \\
\hline Relative risk CTX vs. 2 dose IPTp-SP & 0.72 & Normal(0.72,0.05) & [19] \\
\hline
\end{tabular}

ART antiretroviral therapy for HIV, CTX cotrimoxazole, DALY disability-adjusted life year, IPTp-SP intermittent preventive treatment with sulfadoxine-pyrimethamine

${ }^{\text {a }}$ Labor cost calculations assume that maximum hours of work is $48 \mathrm{~h}$ per week, 4.3 weeks per month [57]

b Age-adjusted incidence per 1000 women (the range is provided to illustrate low and high risk) under the 2-IPT Low Strategy (the Reference Strategy)

Table 2 Model parameters varied across countries

\begin{tabular}{llll}
\hline Country & $\begin{array}{l}\text { Annual incidence of malaria (per 1000 } \\
\text { women) [25] }\end{array}$ & \% of women receiving IPTp-SP [11] $\begin{array}{l}\text { \% of pregnant women living with HIV } \\
\text { who received ART [58] }\end{array}$ \\
\hline Ghana & 127 & 58.2 & 62 \\
Kenya & 63 & 35.5 & 63 \\
Malawi & 174 & 80.7 & 79 \\
Mozambique & 262 & 31.4 & 84 \\
Tanzania & 32 & 58.4 & 73 \\
\hline
\end{tabular}

ART antiretroviral therapy for HIV, IPTp-SP intermittent preventive treatment with sulfadoxine-pyrimethamine

In all simulated strategies, it was assumed that all women were eligible to receive Option $\mathrm{B}+$, the current recommended strategy for prevention of mother-tochild HIV transmission [28]. Under Option B+, all HIVinfected pregnant women receive ART regardless of their CD4 cell counts.

\section{Treatment effectiveness}

To estimate the effectiveness of 2 versus 3 IPTp-SP doses, published meta-analytic estimates were used for the reduction in the incidence of primary health outcomes, based on synthesized data from seven trials in six countries [29]. The outcomes included in our analysis were maternal anaemia (haemoglobin $<60 \mathrm{~g} / \mathrm{L}$ ), maternal malaria parasitaemia (blood films found to be positive for Plasmodium parasites), and low birth weight (LBW $<2500 \mathrm{~g})$. The morbidity and mortality associated with each outcome were used to estimate the disabilityadjusted life year (DALY) implications of each complication based on estimates from a systematic analysis done for the Global Burden of Disease Study [30-33]. For CTX compared to IPTp-SP, treatment effect estimates of the same outcomes were used based on a meta-analysis and a study of HIV-infected pregnant women in Malawi [19, 34].

Compared to 2 doses of IPTp-SP, the relative risk estimates of LBW, anaemia, and malaria parasitaemia for 3 doses of IPTp-SP were 0.86 (95\% CI 0.53-1.39), 0.96 (95\% CI 0.87-1.07), and 0.26 (95\% CI 0.15-0.46), and for CTX were 1.1 (95\% CI $0.86-1.34$ ), 0.72 (95\% CI $0.61-0.83$ ), and 0.43 (95\% CI $0.19-1.10)$, respectively (Tables 1,2 )
[29]. In addition, HIV-infected women receiving both CTX and ART had lower mortality compared to women on ART without CTX when their CD4 cell counts were $<350$ [35]; this mortality benefit was varied in sensitivity analysis.

\section{Costs and utilities}

The treatment (drug) cost, healthcare labor cost, and household costs were included. Healthcare labor costs included the value of nurses' time to administer IPTp-SP and/or CTX assuming estimated labour time per IPTpSP administration and nurse's monthly cost of labour. Household costs included direct costs of antenatal care clinic visits and indirect costs of transportation and time spent travelling to and waiting at the health facility. Due to lack of available data on costs associated specific health outcomes modeled in this study, cost savings from averting the malaria complications were not included. All costs were converted to 2015 US dollars using the Consumer Price Index [36].

Health effects were tallied in terms of DALYs averted for the primary health outcomes (LBW, anaemia, and malaria parasitaemia) between competing treatment strategies. The primary outcome of the analysis was the cost per DALY averted. Disutility of disease states to calculate DALYs were based on estimates from the Global Burden of Disease Study and WHO [31-33]. Incremental cost per DALY averted were calculated between competing treatment strategies. All costs and DALYs were discounted at $3 \%$ annual rates [37]. A willingness to pay (WTP) of less than each country's gross domestic 
product (GDP) per capita was used as a threshold to determine cost-effectiveness [38].

\section{Sensitivity analyses}

One-way sensitivity analyses were performed to investigate the effects of changes in model parameters on estimated outcomes across a wide range of values, including risk of the three primary health outcomes, CTX effectiveness, and adherence to daily CTX. In the base case scenario, CTX was significantly more effective than IPTp-SP in reducing the risk of anaemia and malaria parasitaemia; two-way sensitivity analysis was conducted on the risk of anaemia and malaria parasitaemia to assess sensitivity of results to changes in CTX effectiveness.

In the base case scenario, pregnant women experienced lower mortality from receiving CTX and ART concurrently than receiving ART only when their CD4 cell counts were $<350$ cells $/ \mathrm{mm}^{3}$. These mortality benefits were excluded in sensitivity analysis to isolate the benefits associated with the three primary health outcomes.

IPTp-SP is recommended starting as early as possible in the second trimester while CTX can be administered during the first trimester. In sensitivity analysis, the impact of initiating CTX therapy in the second trimester and how the benefits from CTX would be expected to change relative to the 3-IPT High Strategy when the CTX benefits start to accrue at the same time as IPTp-SP were examined.

Additionally, a probabilistic sensitivity analysis was conducted. For each scenario, the model was re-run 10,000 times while repeatedly Monte Carlo sampling from the probability distributions of all input parameters (Tables 1,2), generating 95\% confidence intervals around all outcomes as per ISPOR guidelines [39]. All analyses were performed in $R$ (version 3.2.1, The $\mathrm{R}$ Foundation for Statistical Computing, Vienna) using the computer code linked in the Additional file 1 [40-43].

\section{Role of the funding source}

The funders of the study had no role in study design, data collection, data analysis, data interpretation, or writing of the report. The corresponding author had full access to all the data in the study and had final responsibility for the decision to submit for publication.

\section{Results}

\section{Base case results}

Figure 1 shows projected reductions in the primary outcomes (LBW, anaemia, and malaria parasitaemia) for each strategy relative to the Reference Strategy in each of the studied countries. Both the CTX and 3-IPT High Strategies substantially reduced the incidence of the three health outcomes in all countries compared to the

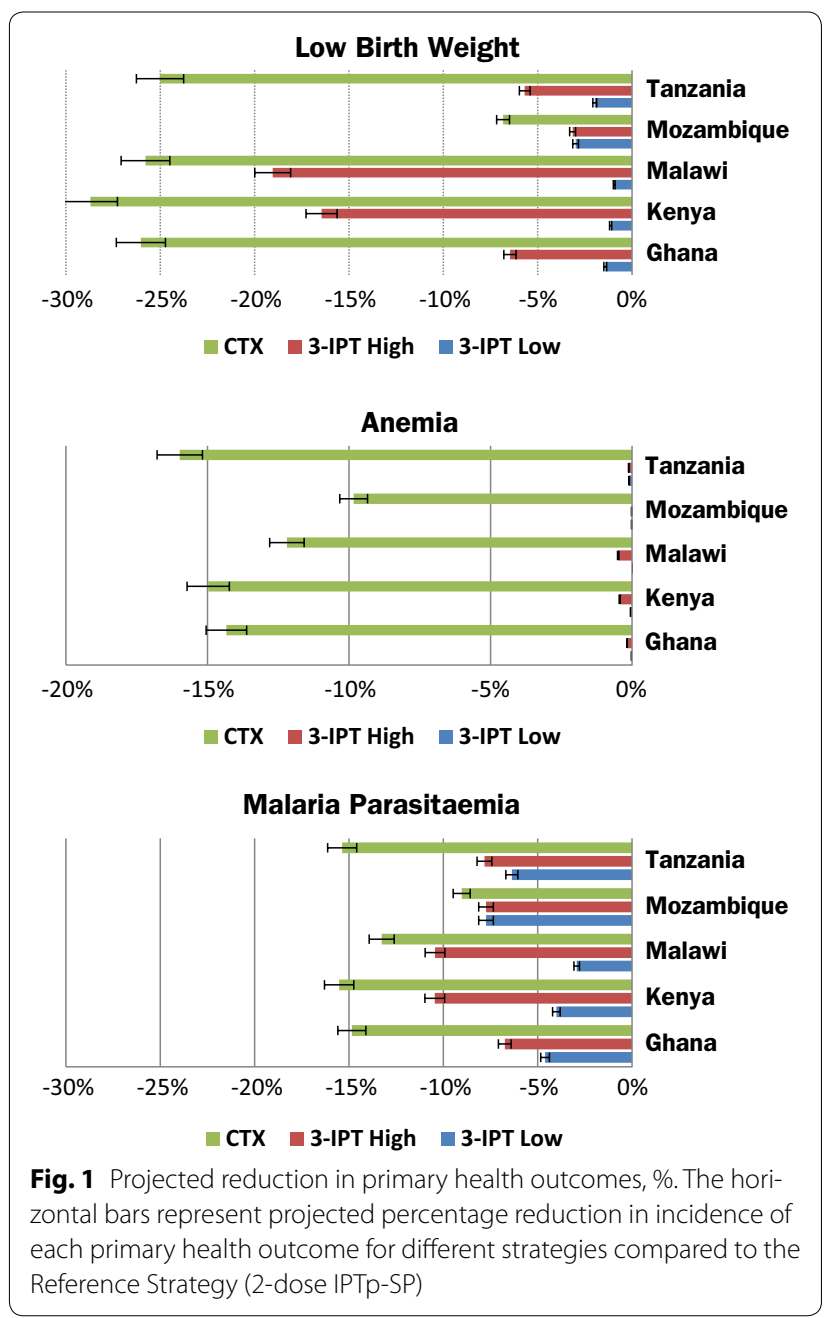

Reference Strategy. The largest reductions accrued from the CTX Strategy (partly due to the preventive benefits in the first trimester, tested in sensitivity analysis below), and relatively higher coverage rate of CTX compared to IPT in low coverage strategies). Compared to the Reference Strategy, the CTX Strategy reduced incidence of LBW by $6.8 \%$ (95\% CI $4.2-9.4 \%$ ) to $28.7 \%$ (95\% CI $21.7-$ 35.7 ), anaemia by $9.8 \%$ (95\% CI $7.2-12.4$ ) to $14.5 \%$ ( $95 \%$ CI $8.0-21.9)$, and malaria parasitaemia by $9.0 \%(95 \%$ CI 6.4-11.6) to $15.5 \%$ (95\% CI 8.5-22.5) across the five countries.

Base case cost-effectiveness results are presented in Table 3. In all countries, the 3-IPT High Strategy was strictly dominated by the CTX Strategy, which averted more DALYs at lower cost. The 3-IPT Low Strategy was eliminated from consideration by extended dominance; the incremental cost-effectiveness ratio (ICER) of the 3-IPT Low Strategy was higher than that of the CTX Strategy compared to the Reference Strategy except in Mozambique. Compared to the Reference Strategy, the 
Table 3 Base case results

\begin{tabular}{|c|c|c|c|c|c|}
\hline Country and strategy & $\begin{array}{l}\text { Costs per 10,000 women } \\
\text { (USD) }\end{array}$ & DALYs per 10,000 women & Incremental cost & Incremental DALYs averted & ICER \\
\hline \multicolumn{6}{|l|}{ Ghana } \\
\hline 2-IPT Low ${ }^{\text {a }}$ & 359,992 & 12,055 & & & \\
\hline 3-IPT Low & 360,340 & 11,912 & 348 & 143 & Dominated $^{b}$ \\
\hline 3-IPT High & 361,594 & 11,434 & 1602 & 621 & Dominated \\
\hline CTX & 361,009 & 9312 & 1017 & 2743 & $\$ 0.37$ \\
\hline \multicolumn{6}{|l|}{ Malawi } \\
\hline 2-IPT Low ${ }^{a}$ & 393,772 & 14,671 & & & \\
\hline 3-IPT Low & 394,744 & 14,563 & 972 & 108 & Dominated $^{b}$ \\
\hline 3-IPT High & 399,172 & 12,382 & 5400 & 2289 & Dominated \\
\hline CTX & 396,580 & 11,317 & 2808 & 3354 & $\$ 0.84$ \\
\hline \multicolumn{6}{|l|}{ Kenya } \\
\hline 2-IPT Low ${ }^{a}$ & 383,217 & 7000 & & & \\
\hline 3-IPT Low & 384,314 & 6934 & 1097 & 66 & Dominated $^{b}$ \\
\hline 3-IPT High & 388,568 & 6183 & 5351 & 817 & Dominated \\
\hline CTX & 386,518 & 5339 & 3301 & 1661 & $\$ 1.99$ \\
\hline \multicolumn{6}{|l|}{ Mozambique } \\
\hline 2-IPT Lowa & 406,628 & 16,424 & & & \\
\hline 3-IPT Low & 409,130 & 16,022 & 2502 & 402 & Dominated \\
\hline 3-IPT High & 409,367 & 15,997 & 2739 & 427 & Dominated \\
\hline CTX & 404,816 & 15,240 & -1812 & 1184 & Cost-saving \\
\hline \multicolumn{6}{|l|}{ Tanzania } \\
\hline 2-IPT Low & 367,892 & 4364 & & & \\
\hline 3-IPT Low & 370,519 & 4327 & 2627 & 37 & Dominated $^{b}$ \\
\hline 3-IPT High & 371,468 & 4231 & 3576 & 133 & Dominated \\
\hline CTX & 371,154 & 3516 & 3262 & 848 & $\$ 3.85$ \\
\hline
\end{tabular}

CTX cotrimoxazole, DALY disability-adjusted life year, ICER incremental cost-effectiveness ratio (cost per DALY averted) compared to the strategy in the row above, IPT intermittent preventive treatment with sulfadoxine-pyrimethamine

a Reference Strategy

b Dominated by extended dominance: a linear combination of two other strategies yields greater benefit at equal cost

CTX Strategy resulted in ICERs ranging from $\$ 0.4$ to $\$ 3.9$ per DALY averted in countries other than Mozambique. In Mozambique, due to relatively higher baseline IPT coverage and higher risk of malaria compared to other studied countries, the 3-IPT Low Strategy was strictly dominated by the CTX Strategy, and as compared to the Reference Strategy, the CTX was cost saving (averting more DALYs at lower cost). DALYs averted from the CTX Strategy compared to any of the IPTp-SP strategies primarily accrued from pregnant women receiving benefits from CTX earlier than IPTp-SP since preventive benefits started during the first trimester for CTX and during the second trimester for IPTp-SP.

\section{Results of sensitivity analyses}

Results of sensitivity analyses on CTX effectiveness in reducing the incidence of the three primary health outcomes are summarized in Fig. 2, which shows ICERs of the CTX Strategy compared to the Reference Strategy.
The effects of results of changes in CTX effectiveness in reducing the incidence of the three primary health outcomes would affect results were assessed. Results were most sensitive to changes in CTX effectiveness in reducing LBW incidence. Compared to the Reference Strategy, when the relative risk estimate of CTX relative to 3 -IPT for LBW increases by $10 \%$ or more (i.e., benefits of CTX decline), the CTX Strategy no longer dominates the 3-IPT High Strategy. Results were not substantially affected by varying the effectiveness of CTX in reducing the incidence of maternal anaemia and malaria parasitaemia over the range of plausible values.

Effects of adherence to daily CTX on health outcomes and cost-effectiveness were evaluated. Perfect adherence to CTX was assumed in the base case. In high-incidence countries (Malawi and Mozambique), when more than $0.5 \%$ of pregnant women did not adhere to CTX every week (thus, more than $18 \%$ of women dropped out over the pregnancy period), the 3-IPT High Strategy became 


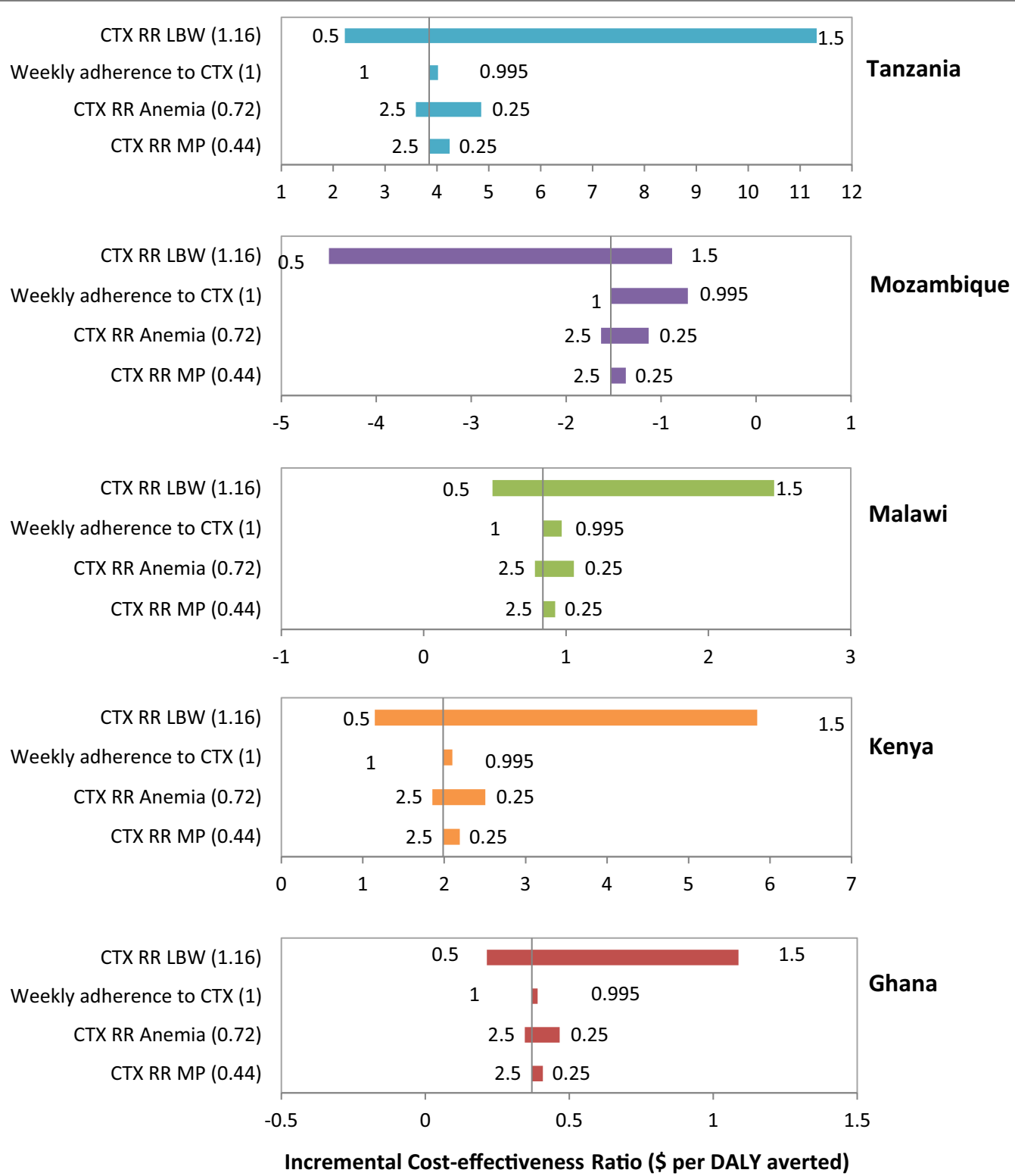

Fig. 2 One-way sensitivity analyses: CTX Strategy compared to Reference Strategy. The vertical lines represents the ICER in the base case analysis (2015 US dollars per DALY averted) and the horizontal bars represent the variation of the ICER given variations of key parameters. The numbers at each end of the bars represent the lower and upper bounds of the value used for each parameter. Numbers in brackets represent the deterministic value of each parameter (CTX RR estimates are compared to 2-dose IPTp-SP). CTX cotrimoxazole, RR relative risk, LBW low birth weight, MP malaria parasitaemia

the preferred strategy, averting more DALYs than the CTX Strategy with ICERs ranging from $\$ 2.6$ to $\$ 13.5$ at varying weekly CTX dropout rates (Additional file 1: Table S1 and Figure S2). In lower-incidence countries, when more than $1.5 \%$ (Kenya) to $2.5 \%$ (Ghana and Tanzania) of pregnant women dropped out weekly (thus, more than 45 and $64 \%$ of women, respectively, dropped out over the pregnancy period), the 3-IPT High Strategy became the preferred strategy.
When mortality benefits were excluded from receiving CTX and ART rather than ART only, the CTX Strategy was still the most effective strategy, averting more DALYs than the 3-IPT High Strategy, and was cost-saving. In this case, the CTX Strategy averted 382 (95\% CI 374-330) more DALYs than the 3-IPT High Strategy per 10,000 pregnant women.

When pregnant women started CTX in the second trimester (rather than in the first trimester as assumed 
in the base case for the CTX Strategy), the 3-IPT High Strategy was more effective and cost-effective than the CTX Strategy, averting 1503 (95\% CI 1495-1511) more DALYs per 10,000 women at a cost of $\$ 7905$ (95\% CI 7866-7944), for an ICER of $\$ 5.3$ (95\% CI 5.2-5.3) per DALY averted.

In probabilistic sensitivity analyses, it was found that at all willingness-to-pay (WTP) thresholds, the CTX Strategy was always preferred. Results for Malawi are shown in Fig. 3. In Malawi, for a WTP < \$2.04 per DALY averted, the 3-IPT Low Strategy was more often costeffective (compared to the Reference Strategy) than the 3-IPT High Strategy. For a WTP greater than $\$ 9.00$ per DALY averted, the 3-IPT High Strategy and CTX Strategy were always cost-effective compared to the Reference Strategy.

\section{Discussion}

Based on the analysis of the study, in regions of sub-Saharan Africa with high HIV and malaria burden, administering daily cotrimoxazole to HIV-infected pregnant women regardless of $\mathrm{CD} 4$ cell count is the preferred strategy under most conditions. The CTX Strategy dominates the 3-IPT High Strategy by averting more DALYs at less cost in most countries. The sensitivity analyses indicate that the CTX Strategy is the most effective strategy over a wide range of assumptions about key parameter values.

The results indicate that daily CTX for HIV-infected pregnant women would generate substantial reductions in the incidence of three primary health outcomesmaternal anaemia, malaria parasitaemia, and LBW-that cause avertable disease burden in pregnant women and

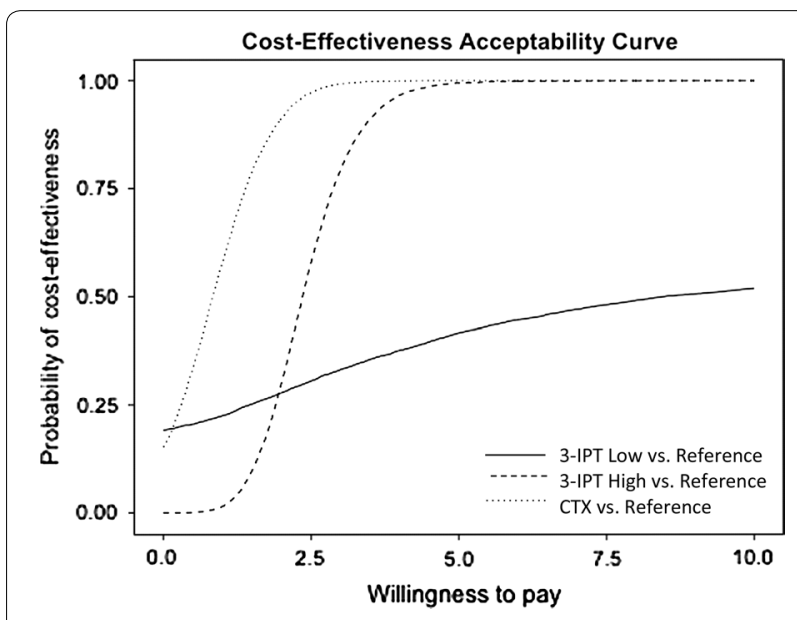

Fig. 3 Cost-effectiveness acceptability curve for Malawi. Willingness to pay (WTP) is expressed in 2015 US dollars per DALY averted. Each line represents the probability of each strategy being cost-effective compared to the Reference Strategy (2-dose IPTp-SP) for different WTP thresholds infants in malaria endemic regions. Additionally, the CTX Strategy yielded ICERs ranging from cost-saving to \$3.9 compared to the Reference Strategy-far below WTP thresholds in the studied countries. These results hold as long the weekly CTX dropout rate is $<0.5 \%$ (equivalent to $18 \%$ over the entire pregnancy period) in high-incidence countries (Malawi and Mozambique) and $<1.5 \%$ ( $45 \%$ over the entire pregnancy period) in low-incidence countries (Tanzania, Kenya, and Ghana). A recent clinical trial found that fewer than $10 \%$ of HIVinfected pregnant women reported incomplete adherence to CTX [44]. Thus, the results of the current study suggest that the CTX Strategy is likely to be the preferred strategy over a reasonable range of incomplete adherence rates [44].

The findings from the study support the updated WHO guidelines that recommend continued use of CTX regardless of CD4 cell count where malaria is common [8]. While the results are contingent upon initiation of CTX earlier in the course of pregnancy and adherence to the daily CTX dose, given currently available data on the efficacy of each prevention strategy and associated cost estimates, CTX is likely to be the preferred strategy. The cost estimates are conservative, as cost savings associated with reduced maternal anaemia, malaria parasitaemia, and LBW were not included due to lack of data on specific breakdown of the costs. If associated cost savings were included, the CTX Strategy would look even more favourable compared to the 3-IPT High Strategy.

This analysis has several limitations. First, we modeled the effects of each strategy based on published meta-analytic data and results from randomized trials $[19,21,29$, 45]. Due to lack of data directly comparing the prevention strategies, results from randomized trial and meta-analytic data were combined, and the relative risks/benefits of each strategy compared to the Reference Strategy were calculated. In addition, the meta-analytic data had some potential sources of bias, which could have led to overestimation of the effectiveness of the 3-IPT strategies [29]. Second, the rate of malaria infection was modeled as a function of CD4 cell count and preventive treatment status, and malaria parasite transmission by mosquitoes was not modeled because the interventions would not differentially affect the transmission dynamics in the homogeneously simulated population, and thus malaria transmission risks. Also, the model was simulated for a short period of time, which was unlikely to have major impact on parasite dynamics. However, inclusion of the dynamics of malaria infection is unlikely to change our results, other than potentially increasing the estimated benefits from the preventive treatment.

While the model results were quite robust to varying levels of model parameters, the lack of head-to-head 
comparisons of CTX and IPTp-SP suggests that a randomized controlled trial assessing the efficacy of CTX compared to 3 doses of IPTp-SP could be informative, particularly in areas with low malaria transmission, and could guide future modeling studies of the cost-effectiveness of these interventions.

\section{Conclusions}

A strategy of providing daily CTX to HIV-infected pregnant women in malaria-endemic regions is generally more effective and less costly than strategies that provide 2 or 3 doses of IPTp-SP. Administering CTX in addition to ART for all HIV-infected pregnant women in such areas, regardless of CD4 count, would not only be costeffective but also feasible to implement.

\section{Additional file}

Additional file 1. Appendix.

\section{Authors' contributions}

SEC: conceived the work, helped with study conceptualization, carried out the analyses, critically assessed the methods and findings, contributed to the preparation of the manuscript; MLB: conceived the work, critically assessed the methods and findings, contributed to the preparation of the manuscript; EB: conceived the work, helped with study conceptualization, critically assessed the methods and findings, contributed to the preparation of the manuscript. All authors read and approved the final manuscript.

\section{Author details}

1 Department of Management Science and Engineering, Stanford University, Stanford, CA, USA. ${ }^{2}$ Division of General Medical Disciplines, Stanford University, Stanford, CA, USA. ${ }^{3}$ Center for Health Policy and the Center for Primary Care and Outcomes Research, Stanford University, Stanford, CA, USA.

\section{Competing interests}

The authors declare that they have no competing interests.

\section{Availability of data and materials}

Data used for this study are listed in Tables 1 and 2.

\section{Ethics approval and consent to participate}

Not applicable.

\section{Source of funding}

Research reported in this publication was supported by the National Institute on Drug Abuse under Grant R01-DA15612.

\section{Publisher's Note}

Springer Nature remains neutral with regard to jurisdictional claims in published maps and institutional affiliations.

Received: 22 May 2017 Accepted: 30 September 2017

Published online: 06 October 2017

\section{References}

1. Institute for Health Metrics and Evaluation. Global Burden of Disease (GBD) Study 2015. http://ghdx.healthdata.org/gbd-2015/data-inputsources. Accessed 12 Mar 2017.
2. UNAIDS. HIV and AIDS estimates. http://www.unaids.org/en/regionscountries/countries/malawi/. Accessed 2 Feb 2016.

3. Chirenda J, Murugasampillay S. Malaria and HIV co-infection: available evidence, gaps and possible interventions. Cent Afr J Med. 2003;49:66-71.

4. Mermin J, Lule JR, Ekwaru JP. Association between malaria and CD4 cell count decline among persons with HIV. J Acquir Immune Defic Syndr. 2006:41:129-30.

5. Uneke CJ, Ogbonna A. Malaria and HIV co-infection in pregnancy in subSaharan Africa: impact of treatment using antimalarial and antiretroviral agents. Trans R Soc Trop Med Hyg. 2009;103:761-7.

6. Perrault SD, Hajek J, Zhong K, Owino SO, Sichangi M, Smith G, et al. Human immunodeficiency virus co-infection increases placental parasite density and transplacental malaria transmission in Western Kenya. Am J Trop Med Hyg. 2009;80:119-25.

7. Verhoeff FH, Brabin BJ, Hart CA, Chimsuku L, Kazembe P, Broadhead RL. Increased prevalence of malaria in HIV-infected pregnant women and its implications for malaria control. Trop Med Int Health. 1999;4:5-12.

8. WHO. Co-trimoxazole prophylaxis for malaria and bacterial infections in people with HIV. Geneva, World Health Organization, 2014. http://www. who.int/hiv/topics/arv/cotrimoxazole_factsheet_dec2014/en/. Accessed 25 Jan 2017.

9. WHO. Malaria and HIV interactions and their implications for public health policy. Geneva, World Health Organization. http://www.who.int/ hiv/pub/prev_care/malaria/en/. Accessed 25 Jan 2017.

10. WHO. Use of antiretroviral drugs for treating pregnant women and preventing HIV infection in infants. Geneva, World Health Organization. http://www.who.int/hiv/PMTCT_update.pdf. Accessed 25 Jan 2017.

11. van Eijk AM, Hill J, Alegana VA, Kirui V, Gething PW, ter Kuile FO, et al. Coverage of malaria protection in pregnant women in sub-Saharan Africa: a synthesis and analysis of national survey data. Lancet Infect Dis. 2011;11:190-207.

12. van Eijk AM, Hill J, Larsen DA, Webster J, Steketee RW, Eisele TP, et al. Coverage of intermittent preventive treatment and insecticide-treated nets for the control of malaria during pregnancy in sub-Saharan Africa: a synthesis and meta-analysis of national survey data, 2009-11. Lancet Infect Dis. 2013;13:1029-42.

13. Chico RM, Dellicour S, Roman E, Mangiaterra V, Coleman J, Menendez C, et al. Global Call to Action: maximize the public health impact of intermittent preventive treatment of malaria in pregnancy in sub-Saharan Africa. Malar J. 2015;14:207.

14. Crawley J, Hill J, Yartey J, Robalo M, Serufilira A, Ba-Nguz A, et al. From evidence to action? Challenges to policy change and programme delivery for malaria in pregnancy. Lancet Infect Dis. 2007;7:145-55.

15. Launiala A, Honkasalo ML. Ethnographic study of factors influencing compliance to intermittent preventive treatment of malaria during pregnancy among Yao women in rural Malawi. Trans R Soc Trop Med Hyg. 2007;101:980-9.

16. Marchant T, Nathan $R$, Jones $C$, Mponda H, Bruce J, Sedekia Y, et al. Individual, facility and policy level influences on national coverage estimates for intermittent preventive treatment of malaria in pregnancy in Tanzania. Malar J. 2008;7:260.

17. Mubyazi GM, Magnussen P, Goodman C, Bygbjerg IC, Kitua AY, Olsen $\mathrm{OE}$, et al. Implementing intermittent preventive treatment for malaria in pregnancy: review of prospects, achievements, challenges and agenda for research. Open Trop Med J. 2008;1:92-100.

18. Ndyomugyenyi R, Katamanywa J. Intermittent preventive treatment of malaria in pregnancy (IPTp): do frequent antenatal care visits ensure access and compliance to IPTp in Ugandan rural communities? Trans $R$ Soc Trop Med Hyg. 2010;104:536-40.

19. Kapito-Tembo A, Meshnick SR, van Hensbroek MB, Phiri K, Fitzgerald M, Mwapasa $\vee$. Marked reduction in prevalence of malaria parasitemia and anemia in HIV-infected pregnant women taking cotrimoxazole with or without sulfadoxine-pyrimethamine intermittent preventive therapy during pregnancy in Malawi. J Infect Dis. 2011;203:464-72.

20. Thera MA, Sehdev PS, Coulibaly D, Traore K, Garba MN, Cissoko Y, et al. Impact of trimethoprim-sulfamethoxazole prophylaxis on falciparum malaria infection and disease. J Infect Dis. 2005;192:1823-9.

21. Mermin J, Ekwaru JP, Liechty CA, Were W, Downing R, Ransom R, et al. Effect of co-trimoxazole prophylaxis, antiretroviral therapy, and insecticide-treated bednets on the frequency of malaria in HIV-1-infected adults in Uganda: a prospective cohort study. Lancet. 2006;367:1256-61. 
22. Manyando C, Njunju EM, D'Alessandro U, Van Geertruyden JP. Safety and efficacy of co-trimoxazole for treatment and prevention of Plasmodium falciparum malaria: a systematic review. PLoS ONE. 2013;8:e56916.

23. WHO. Intermittent preventive treatment in pregnancy (IPTp). Geneva, World Health Organization. http://www.who.int/malaria/areas/preventive_therapies/pregnancy/en/. Accessed 5 Jan 2017.

24. Caro JJ, Briggs AH, Siebert U, Kuntz KM. Force I-SMGRPT. Modeling good research practices-overview: a report of the ISPOR-SMDM Modeling Good Research Practices Task Force-1. Med Decis Mak. 2012;32:667-77.

25. WHO. Malaria Country profiles. Geneva, World Health Organization, 2015. http://www.who.int/malaria/publications/country-profiles/en/. Accessed 5 Jan 2016.

26. Gomez PP, Gutman J, Roman E, Dickerson A, Andre ZH, Youll S, et al. Assessment of the consistency of national-level policies and guidelines for malaria in pregnancy in five African countries. Malar J. 2014;13:212.

27. Ford N, Shubber Z, Jao J, Abrams EJ, Frigati L, Mofenson L. Safety of cotrimoxazole in pregnancy: a systematic review and meta-analysis. J Acquir Immune Defic Syndr. 2014;66:512-21.

28. WHO. Antiretroviral drugs for treating pregnant women and preventing HIV infections in infants. Geneva, World Health Organization. http://www. who.int/hiv/pub/mtct/PMTCTfactsheet/en/. Accessed 2 May 2016.

29. Kayentao K, Garner P, van Eijk AM, Naidoo I, Roper C, Mulokozi A, et al. Intermittent preventive therapy for malaria during pregnancy using 2 vs 3 or more doses of sulfadoxine-pyrimethamine and risk of low birth weight in Africa: systematic review and meta-analysis. JAMA. 2013;309:594-604.

30. Fernandes S, Sicuri E, Kayentao K, van Eijk AM, Hill J, Webster J, et al. Cost-effectiveness of two versus three or more doses of intermittent preventive treatment for malaria during pregnancy in sub-Saharan Africa: a modelling study of meta-analysis and cost data. Lancet Glob Health. 2015;3:e143-53.

31. Salomon JA, Vos T, Hogan DR, Jonas JB. Common values in assessing health outcomes from disease and injury: disability weights measurement study for the Global Burden of Disease Study 2010 (vol 380, pg 2129, 2012). Lancet. 2013;381:628.

32. Wang H, Dwyer-Lindgren L, Lofgren KT, Rajaratnam JK, Marcus JR, LevinRector A, et al. Age-specific and sex-specific mortality in 187 countries, 1970-2010: a systematic analysis for the Global Burden of Disease Study 2010. Lancet. 2012;380:2071-94.

33. WHO. Global burden of disease 2004 update: disability weights for diseases and conditions. Geneva, World Health Organization. http://www. who.int/healthinfo/global_burden_disease/GBD2004_DisabilityWeights. pdf. Accessed 25 Jan 2017.

34. Suthar AB, Vitoria MA, Nagata JM, Anglaret X, Mbori-Ngacha D, Sued $\mathrm{O}$, et al. Co-trimoxazole prophylaxis in adults, including pregnant women, with HIV: a systematic review and meta-analysis. Lancet HIV. 2015;2:e137-50.

35. Lowrance D, Makombe S, Harries A, Yu J, Aberle-Grasse J, Eiger O, et al. Lower early mortality rates among patients receiving antiretroviral treatment at clinics offering cotrimoxazole prophylaxis in Malawi. J Acquir Immune Defic Syndr. 2007;46:56-61.

36. Bureau of Labor Statistics. Consumer Price Index (CPI) inflation calculator. http://www.bls.gov/data/inflation_calculator.htm. Accessed 5 Jan 2016.

37. Sanders GD, Neumann PJ, Basu A, Brock DW, Feeny D, Krahn M, et al. Recommendations for conduct, methodological practices, and reporting of cost-effectiveness analyses: second panel on cost-effectiveness in health and medicine. JAMA. 2016;316:1093-103.

38. Murray CJ, Lopez A. World Health Report 2002: reducing risks, promoting healthy life. Geneva: World Health Organization; 2002. p. 186.

39. Briggs $A H$, Weinstein MC, Fenwick EA, Karnon J, Sculpher MJ, Paltiel AD, et al. Model parameter estimation and uncertainty analysis: a report of the ISPOR-SMDM Modeling Good Research Practices Task Force Working Group-6. Med Decis Mak. 2012;32:722-32.

40. Warnes GR, Bolker B, Gorjanc G, Grothendieck G, Korosec A, Lumley T, et al. gdata: Various R Programming Tools for Data Manipulation. $R$ package version 2.18.0. https://cran.r-project.org/package=gdata. Accessed 21 Sept 2017.

41. Genz A, Bretz F. Computation of multivariate normal and t probabilities. Berlin: Springer-Verlag; 2009.

42. Genz A, Bretz F, Miwa T, Mi X, Leisch F, Scheipl F, Hothorn T. mvtnorm: multivariate normal and t distributions. R package version 1.0-6. http:// cran.r-project.org/package=mvtnorm. Accessed 21 Sept 2017.

43. Venables WN, Ripley BD. Modern applied statistics with S. 4th ed. New York: Springer; 2002.

44. Denoeud-Ndam L, Zannou DM, Fourcade C, Taron-Brocard C, Porcher R, Atadokpede F, et al. Cotrimoxazole prophylaxis versus mefloquine intermittent preventive treatment to prevent malaria in HIV-infected pregnant women: two randomized controlled trials. J Acquir Immune Defic Syndr. 2014;65:198-206.

45. Gutman J, Mwandama D, Wiegand RE, Ali D, Mathanga DP, Skarbinski J. Effectiveness of intermittent preventive treatment with sulfadoxinepyrimethamine during pregnancy on maternal and birth outcomes in Machinga district, Malawi. J Infect Dis. 2013;208:907-16.

46. ter Kuile FO, Parise ME, Verhoeff FH, Udhayakumar V, Newman RD, van Eijk AM, et al. The burden of co-infection with human immunodeficiency virus type 1 and malaria in pregnant women in sub-Saharan Africa. Am J Trop Med Hyg. 2004;71:41-54.

47. Liotta G, Mancinelli S, Nielsen-Saines K, Gennaro E, Scarcella P, Magid NA, et al. Reduction of maternal mortality with highly active antiretroviral therapy in a large cohort of HIV-infected pregnant women in Malawi and Mozambique. PLoS ONE. 2013;8:e71653.

48. Centers for Disease Control and Prevention. Interaction of HIV and malaria. http://www.cdc.gov/malaria/ppt/Malaria_HIV_Rick_website.ppt. Accessed 25 Jan 2017.

49. Calmy A, Balestre E, Bonnet F, Boulle A, Sprinz E, Wood R, et al. Mean CD4 cell count changes in patients failing a first-line antiretroviral therapy in resource-limited settings. BMC Infect Dis. 2012;12:147.

50. Zaba B, Calvert C, Marston M, Isingo R, Nakiyingi-Miiro J, Lutalo T, et al. Effect of HIV infection on pregnancy-related mortality in sub-Saharan Africa: secondary analyses of pooled community-based data from the network for Analysing Longitudinal Population-based HIV/AIDS data on Africa (ALPHA). Lancet. 2013;381:1763-71.

51. Schackman BR, Goldie SJ, Freedberg KA, Losina E, Brazier J, Weinstein MC. Comparison of health state utilities using community and patient preference weights derived from a survey of patients with HIV/AIDS. Med Decis Mak. 2002;22:27-38.

52. Price RN, Simpson JA, Nosten F, Luxemburger C, Hkirjaroen L, ter Kuile $F$, et al. Factors contributing to anemia after uncomplicated falciparum malaria. Am J Trop Med Hyg. 2001;65:614-22.

53. Management Sciences for Health. International drug price indicator guide. http://erc.msh.org/mainpage.cfm?file=1.0.htm\&module=DMP\&la nguage=English. Accessed 2 May 2016.

54. Ryan M, Griffin S, Chitah B, Walker AS, Mulenga V, Kalolo D, et al. The costeffectiveness of cotrimoxazole prophylaxis in HIV-infected children in Zambia. Aids. 2008;22:749-57.

55. Granich R, Kahn JG, Bennett R, Holmes CB, Garg N, Serenata C, et al. Expanding ART for treatment and prevention of HIV in South Africa: estimated cost and cost-effectiveness 2011-2050. PLoS ONE. 2012;7:e30216.

56. Klement E, Pitche P, Kendjo E, Singo A, D'Almeida S, Akouete F, et al. Effectiveness of co-trimoxazole to prevent Plasmodium falciparum malaria in HIV-positive pregnant women in sub-Saharan Africa: an open-label, randomized controlled trial. Clin Infect Dis. 2014;58:651-9.

57. Malawi Goverment Employment Act 2000. http://www.ilo.org/wcmsp5/ groups/public/-ed_protect/-protrav/-+ilo_aids/documents/legaldocument/wcms_125534.pdf. Accessed 5 Jan 2016.

58. UNAIDS. Global AIDS Response Progress Report (GARPR)—Malawi Progress Report for 2013. http://www.unaids.org/sites/default/files/country/ documents/MWI_narrative_report_2014.pdf. Accessed 25 Jan 2017. 